\title{
Impact of early initiation versus national standard of care of antiretroviral therapy in Swaziland's public sector health system: study protocol for a stepped-wedge randomized trial
}

Fiona J. Walsh ${ }^{1 *}$, Till Bärnighausen ${ }^{2,3,4}$, Wim Delva ${ }^{5,6,7,8}$, Yvette Fleming ${ }^{9}$, Gavin Khumalo ${ }^{10}$, Charlotte L. Lejeune ${ }^{11}$, Sikhathele Mazibuko ${ }^{12}$, Charmaine Khudzie Mlambo ${ }^{11}$, Ria Reis ${ }^{13,14,15}$, Donna Spiegelman ${ }^{2}$, Mandisa Zwane ${ }^{16}$ and Velephi Okello ${ }^{12}$

\begin{abstract}
Background: There is robust clinical evidence to support offering early access to antiretroviral treatment (ART) to all HIV-positive individuals, irrespective of disease stage, to both improve patient health outcomes and reduce HIV incidence. However, as the global treatment guidelines shift to meet this evidence, it is still largely unknown if early access to ART for all (also referred to as "treatment as prevention" or "universal test and treat") is a feasible intervention in the resource-limited countries where this approach could have the biggest impact on the course of the HIV epidemics. The MaxART Early Access to ART for All (EAAA) implementation study was designed to determine the feasibility, acceptability, clinical outcomes, affordability, and scalability of offering early antiretroviral treatment to all HIV-positive individuals in Swaziland's public sector health system.

Methods: This is a three-year stepped-wedge randomized design with open enrollment for all adults aged 18 years and older across 14 government-managed health facilities in Swaziland's Hhohho Region. Primary endpoints are retention and viral suppression. Secondary endpoints include ART initiation, adherence, drug resistance, tuberculosis, HIV disease progression, patient satisfaction, and cost per patient per year.

Sites are grouped to transition two at a time from the control (standard of care) to intervention (EAAA) stage at each four-month step. This design will result in approximately one half of the total observation time to accrue in the intervention arm and the other half in the control arm. Our estimated enrolment number, which is supported by conservative power calculations, is 4501 patients over the course of the 36-month study period.

A multidisciplinary, mixed-methods approach will be adopted to supplement the randomized controlled trial and meet the study aims. Additional study components include implementation science, social science, economic evaluation, and predictive HIV incidence modeling.

Discussion: A stepped-wedge randomized design is a causally strong and robust approach to determine if providing antiretroviral treatment for all HIV-positive individuals is a feasible intervention in a resource-limited, public sector health system. We expect our study results to contribute to health policy decisions related to the HIV response in Swaziland and other countries in sub-Saharan Africa.

(Continued on next page)
\end{abstract}

\footnotetext{
* Correspondence: fwalsh@clintonhealthaccess.org

${ }^{1}$ Clinton Health Access Initiative, Boston, MA, USA

Full list of author information is available at the end of the article
} 
(Continued from previous page)

Trial registration: ClinicalTrials.gov, NCT02909218. Registered on 10 July 2016.

Keywords: Antiretroviral treatment, Swaziland, HIV/AIDS, Prevention

\section{Background}

The global community has made extraordinary strides in scaling up treatment for people living with HIV/AIDS over the past two decades. Since 2000, there has been a 30 -fold increase in people accessing antiretroviral therapy (ART) globally from 250,000 people to more than 17 million people in $2015[1,2]$. The rate of new infections globally has also come down in the last decade from 3.3 million new infections in 1998 to 2.1 million in 2015 [2]. This success has been driven, in part, by an unprecedented increase in financial resources, with annual funding levels increasing from US\$5 billion in 2003 to US\$19.2 billion in 2014 [3].

However, despite the significant progress, the global community is still far from ensuring that all people living with HIV/AIDS (PLHIV) eligible for treatment are receiving it. Moreover, without a significant reduction in new infections, the number of individuals in need of treatment will continue to expand, as will the costs of HIV treatment programs $[4,5]$. Identifying and implementing effective prevention interventions to try and get ahead of the epidemic is a crucial next step in the global response to HIV.

Over the past several years, HIV research has demonstrated that earlier and expanded access to ART could have a significant impact on HIV incidence. Results from the HIV Prevention Trials Network 052 (HPTN 052) trial showed that early access to ART prevents onward transmission of the virus to the uninfected partner in heterosexual HIV-discordant couples. In 2011, the trial reported not only a $96 \%$ decrease in HIV transmission, but also a $41 \%$ decrease in HIV-related morbidity from early initiation of ART [6]. The results from the Strategic Timing of Antiretroviral Therapy (START) trial in 2015 demonstrated that the immediate initiation of ART was beneficial for morbidity and mortality with no increased rate of adverse effects [7]. In response to this growing body of epidemiological evidence, World Health Organization (WHO) 2015 treatment guidelines recommended ART initiation for all HIV-positive individuals [8].

While the clinical impact of Early Access to ART for All (EAAA) is evident, critical implementation questions remain. Recently, results from the Treatment as Prevention (TasP) Trial in rural KwaZulu-Natal in South Africa did not demonstrate a difference in HIV incidence between communities randomized to either immediate offer of ART for all HIV-positive individuals compared to the standard of care [9]. The study results demonstrated not only the need for high rates of linkages to care following diagnosis, but also the importance of understanding how to best deliver early ART in southern Africa. As the latest WHO guidelines are recommending EAAA [10] and countries in sub-Saharan Africa start to follow this recommendation [11], the critical question is no longer whether EAAA should be implemented, but rather how to implement it in resourcelimited settings where the epidemic is most prevalent.

It remains unknown, however, what the impact of EAAA policies will be on public sector health systems in sub-Saharan Africa. The overall HIV treatment program effectiveness in successfully treating HIV patients could change, as could patient satisfaction, patients' economic welfare, and provider satisfaction. Policymakers in high HIV prevalence countries and stakeholders in the international community require empirical evidence to assess the feasibility and acceptability of EAAA policies, as well as the clinical outcomes, affordability, and scalability of national implementation of this intervention through routine, public sector health systems.

This study was designed in response to questions posed by Swaziland's policymakers about the impact of an EAAA policy on their national HIV/AIDS care and treatment program. The study aims to generate the evidence needed to more fully understand what is required to successfully implement an EAAA strategy in a public sector health system in sub-Saharan Africa.

\section{Specific aims}

This trial aims to:

1. quantify the causal impact of early access to ART for all HIV-infected adults, irrespective of CD4 count or clinical staging, on ART retention and viral suppression in a public sector health system;

2. quantify the causal impacts of EAAA on average health care expenditures and resources use;

3. quantify the causal impacts of EAAA on patient satisfaction, patients' welfare, and provider satisfaction;

4. to establish the feasibility and acceptability of EAAA;

5. establish the role of PLHIV, traditional leaders, civil society, and communities for implementing EAAA;

6. determine the social and institutional processes throughout EAAA implementation to help interpret differences in health service delivery and patient experiences of ART initiation, adherence, and retention before and after the intervention; 
7. estimate the potential change in HIV incidence and ART program size if EAAA were to be implemented nationwide in Swaziland; and

8. predict the long-term cost-effectiveness of EAAA.

\section{Methods}

\section{Study design}

This is a randomized seven-stepped-wedge design on seven pairs (13 government clinics and one regional hospital) in Hhohho Region (see Fig. 1) with open enrollment for all adults aged $\geq 18$ years.

The study sites include a mix of high-volume and lowvolume facilities (volume of patients) that are already providing a comprehensive package of HIV care and treatment services per Swaziland's national adult HIV treatment guidelines [12].

Sites are grouped to transition two at a time from the control (national eligibility guidelines or current standard of care) to the intervention (EAAA) stage every four months. As illustrated in Fig. 2, the study will enroll all eligible HIV-positive patients who arrive at the facility in Month 1. All sites will start in the standard of care stage ("C"), and then each site will have a four-month transition period ("T") to transition consecutively until all sites are in the intervention ("I") stage. The sites will start implementing the intervention on the first day of the transition period.

Individuals who arrive at one of the study facilities during the control ("C") stage will be offered ART according to the current national guidelines. Sites will follow the current national standard of care for HIV care and treatment while in the control stage.

During the transition period ("T") and the intervention ("I") stage, all HIV-positive patients will be offered ART per the EAAA intervention (an offer of immediate ART

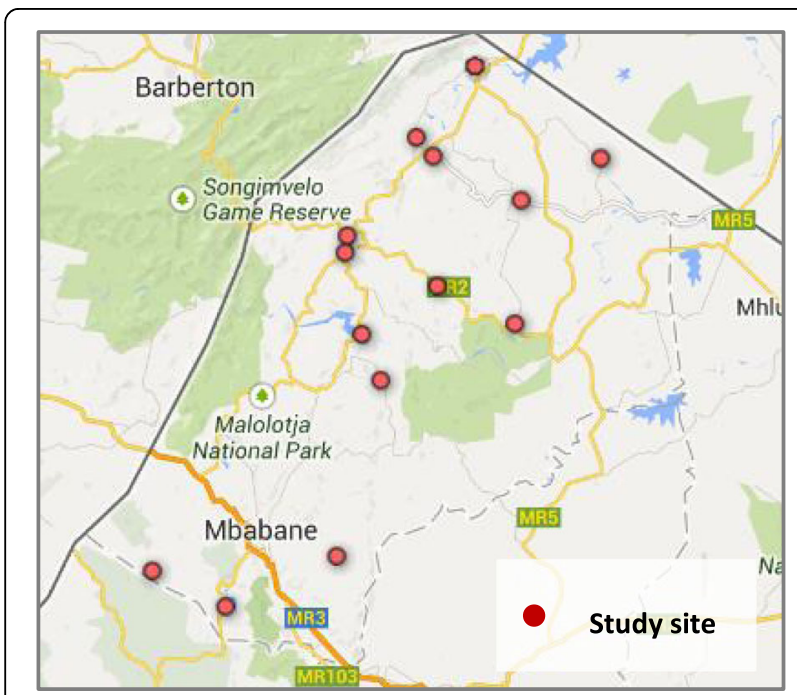

Fig. 1 Map of study sites in Hhohho Region initiation irrespective of CD4). Study participants who were already in pre-ART at the start of the study or who were enrolled into pre-ART during the control stage according to national standard of care will be offered ART at their first follow-up appointment at their enrollment site following the site's transition to the intervention stage. This way, everyone who is enrolled into the study population will eventually be offered the EAAA intervention (Fig. 3).

An additional file provides the protocol checklist (see Additional file 1. SPIRIT checklist for the MaxART EAAA Trial, indicating which manuscript page contains each element of the study protocol).

\section{Setting}

Swaziland faces a prevalence rate of $31-32 \%$ among its 18-49-year-olds, but the epidemic disproportionately affects women [13]. The recent Swaziland HIV Incidence Measurement Survey highlighted that overall HIV incidence is higher among women $(3.1 \%$ for women and $1.7 \%$ for men) and highest of all in women $20-24$ years of age (4.2\%) [13].

The Swaziland National AIDS Program (SNAP) has been in existence since 2004 and has scaled up its program each year, resulting in a significant increase in the number of people on treatment. The country has made great strides in their HIV response, including a robust national ART program and expanding the immunological treatment eligibility criteria in their national HIV treatment guidelines since 2010 to individuals with CD4 $<350$ cells $/ \mathrm{mm}^{3}$. In December 2015, the country further expanded its eligibility criteria to CD $4<500$ cells $/ \mathrm{mm}^{3}$ as per the WHO 2015 guidelines. At the end of December $2015,147,274$ adults were on treatment, which represents more than $90 \%$ treatment coverage based on current eligibility criteria (CD4 count $>500 \quad$ cell $\left./ \mathrm{mm}^{3}\right)$ and estimates of need [14].

\section{Trial participants (inclusion and exclusion criteria)}

All HIV-positive individuals who are aged 18 years or older and are ART naïve-excluding pregnant or breastfeeding women-who attend the health facilities included in the study will be asked for their verbal consent to enroll in the study. Those individuals whose CD4 is greater than the current national guidelines for ART initiation and whose WHO stage is not 3 or 4 are the primary study population. Written informed consent will be secured for participation in social science research and the economic evaluation.

Participants must be able and willing to give verbal consent for trial participation. Individuals considered unable to provide and participate in informed consent include those with uncontrolled psychiatric disorders or severe neurological impairment. 


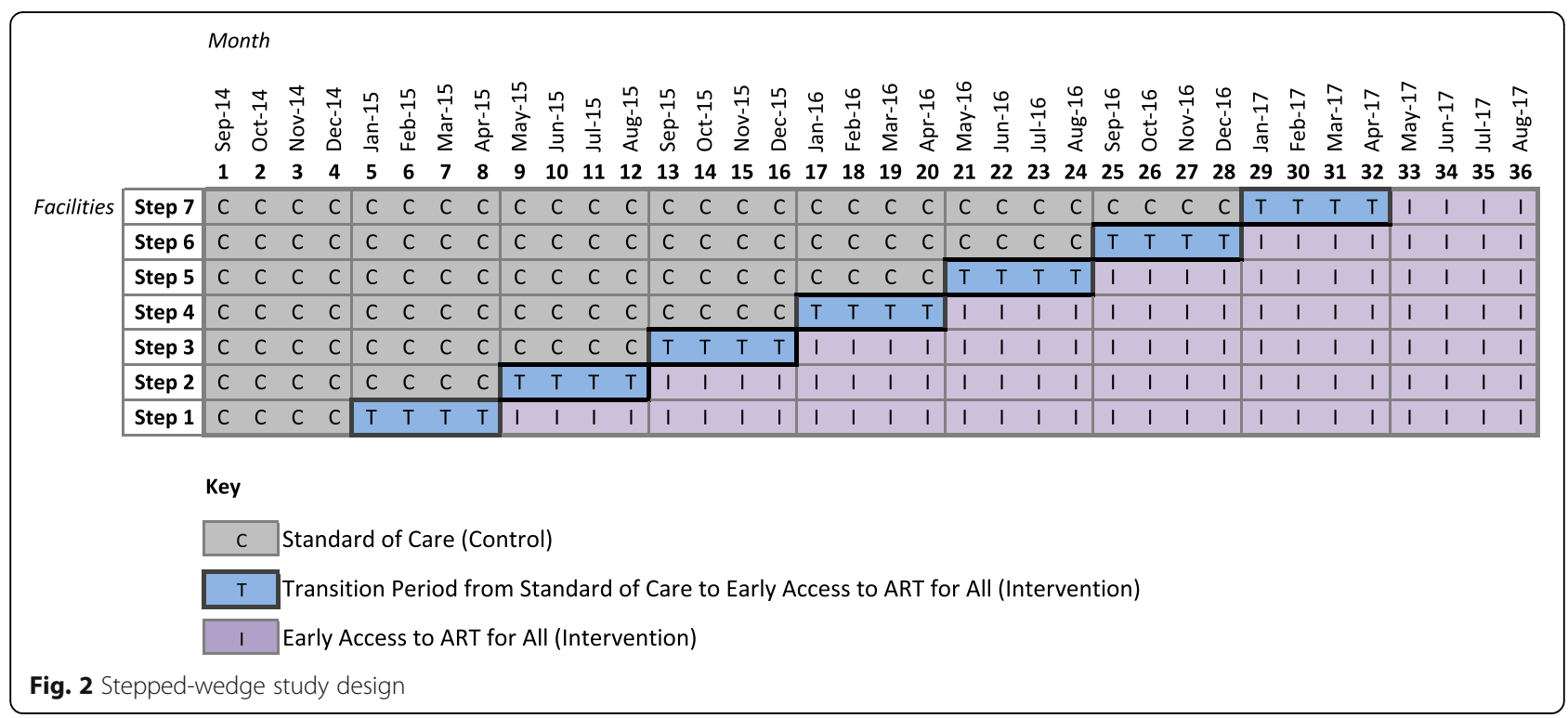

\section{Sample size}

The primary study population will be 2008 newly diagnosed or returning pre-ART patients as defined by the current national guidelines who are enrolled during the control or the intervention stage over the 36-month study period. In addition, 2493 patients with CD4 $\leq 350$ cells $/ \mathrm{mm}^{3}$ will be enrolled to form a total study population of 4501. With recent changes in Swaziland's national guidelines, the number of patients enrolled within the primary population is expected to be lower than projected due to the change in ART initiation threshold.
The trial statisticians performed randomization of the study sites before the start of the study. The 14 sites have been grouped into seven pairs: four pairs were grouped according to geographic proximity; two pairs were grouped to coordinate the timing with operational or logistical issues; and one pair was grouped and excluded from the randomization so that it can transition to the intervention first to accommodate the social science research timeline. The six remaining pairs will be randomized into steps 2 to 7 when each site transitions to the intervention stage, but the health workers,

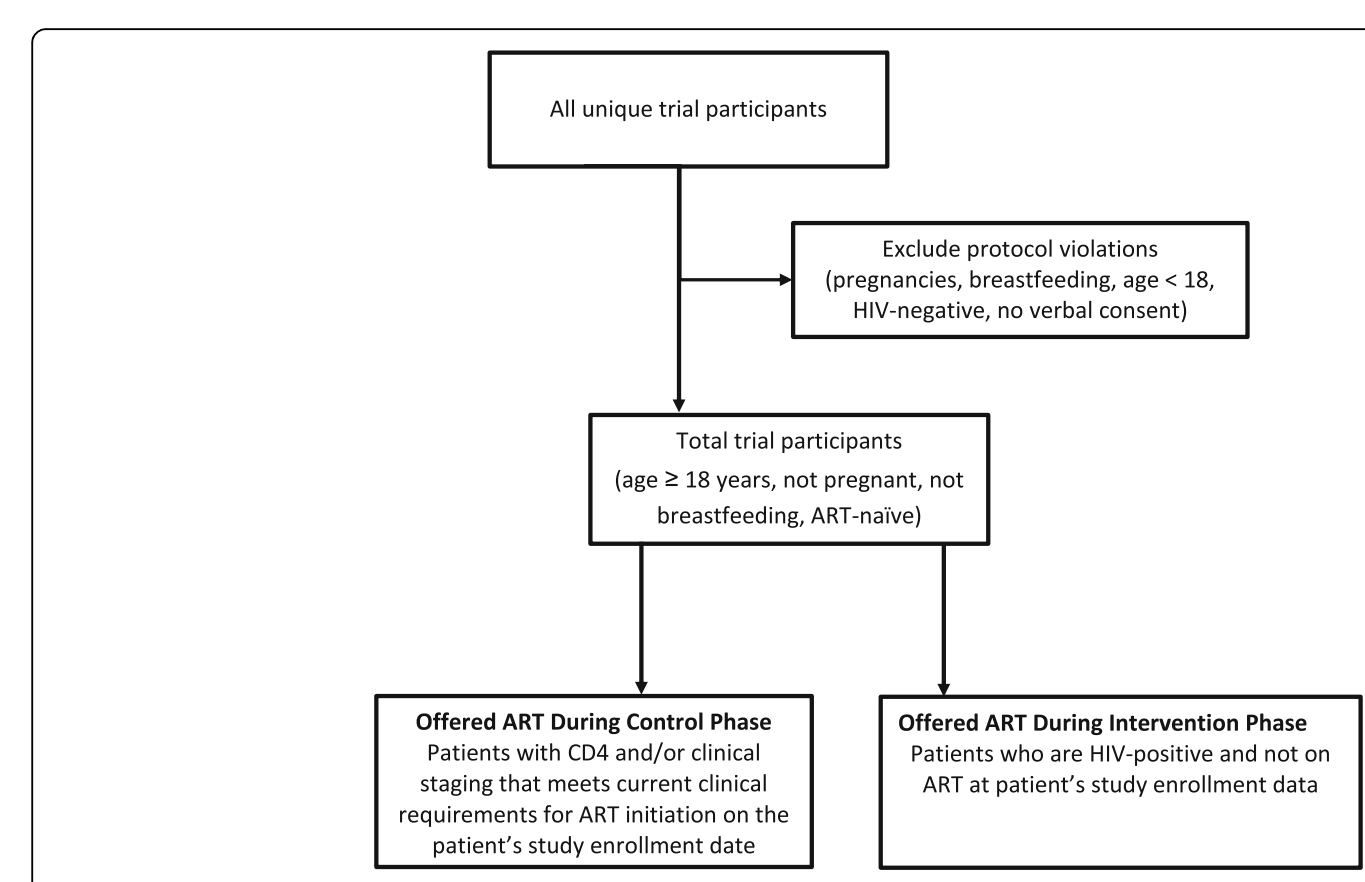

Fig. 3 Trial flow diagram 
study participants, and study teams are blinded to the timing to minimize bias.

\section{Power calculations}

Power calculations were undertaken to ensure that the minimal detectable difference in the two primary endpoint rates, given that the projected sample size was well within the expected given prior literature [15]. For one-year retention, 1160 patients in the primary study population were projected to enroll during the EAAA (intervention) phase and 2088 under the control phase in the first 24 months to achieve a one-year follow-up.

Using the standard method for power calculations for binary endpoints in stepped-wedge designs, with a $5 \%$ two-tailed Type I error rate, an $80 \%$ power or more was found to detect a 6-8 percentage point increase in the one-year retention rate over a range of scenarios considered for the one-year retention rate in SOC from 40$80 \%$ and an intra-cluster correlation coefficient (ICC) of $0-0.5$, with no prior data available in Swaziland to allow ICC estimation. It is well-known that the stepped-wedge design is insensitive to the assumed value of the ICC [15]. To be conservative, we considered a wide range. Also, here, the minimum detectable effect sizes did not vary over the range of ICCs considered.

For the six-month viral load suppression after ART initiation endpoint, 1869 patients were projected to enroll in the EAAA (intervention) phase and 1728 in the control phase over the first 30 months to ascertain a sixmonth viral load after ART initiation. Following the methods as above, we found that there was $80 \%$ power or more to detect a 7-8 percentage point increase in the six-month viral suppression rate over a range of scenarios considered for the six-month viral suppression rate in standard of care of $20-70 \%$ and an intra-cluster correlation coefficient of $0.10-0.20$.

\section{Study procedures}

Community sensitization Community mobilization is used to sensitize communities on early ART and create demand for uptake of services. A comprehensive messaging strategy was designed to address the complex personal, cultural, social, and sexual considerations that influence decisions around HIV testing and ART initiation. Messages are delivered through a variety of methods and activities, including sensitization meeting with key community actors, including traditional leaders to gain their support. Community dialogues targeting community members are conducted using edutainment providing HIV testing services for active case finding at the community level.

Door-to-door visits where information, education, and communication materials are delivered by trained community-based volunteers. Community-based HTC is provided during the community events and linkage to care is ensured through working with expert patients and HIV testing partners. Support groups are equipped with skills and tools to provide treatment literacy to PLHIV. These activities are conducted within a 20-30$\mathrm{km}$ radius around each study site.

\section{Clinical procedures for ART initiation and follow-up}

The study aims to promote a continuum of care and to evaluate feasibility of an EAAA strategy within a public sector health system setting. Therefore, the study will be implemented in a public sector health system where there is already an essential package of services available through the current national ART program. The main differences in the package of services delivered in the standard of care vs. the intervention stage of the study are outlined in Table 1 below.

Additional blood investigations will be conducted to inform the measurement of the study endpoint, viral load suppression, but these lab tests will be taken in both the standard of care and intervention groups. Aside from the eligibility criteria and the messaging used during pre-ART counseling sessions, there will be no differences in the package of follow-up services provided to patients between the control and intervention study groups.

Per national guidelines, ART initiation takes place within four weeks of HIV testing or two weeks of enrollment at the trial clinic, unless delayed per clinician's guidance. All ART drugs used in the trial follow the Swaziland adult HIV management guidelines. All individuals who are eligible for treatment will be initiated on Swaziland's recommended first-line ART regimen (TDF + 3TC + EFV), unless contraindicated when recommended alternate regimens will be used per national guidelines. These alternatives are: $\mathrm{TDF}+3 \mathrm{TC}+\mathrm{NVP}$ or $\mathrm{AZT}+3 \mathrm{TC}+\mathrm{NVP}$ (when EFV cannot be used); $\mathrm{ABC}+$ $3 \mathrm{TC}+\mathrm{EFV}$ or AZT + 3TC + EFV (when TDF cannot be used); $\mathrm{ABC}+3 \mathrm{TC}+\mathrm{EFV}$ or $\mathrm{d} 4 \mathrm{~T}+3 \mathrm{TC}+\mathrm{EFV}$ (when AZT cannot be used).

All patients in the control and intervention phase will follow the nationally recommended follow-up schedule for individuals who are on ART or enrolled in pre-ART.

Table 1 Key differences in package of services in control vs. intervention stage for patients enrolled in the EAAA study

\begin{tabular}{lll}
\hline Service & Control & Intervention \\
\hline $\begin{array}{l}\text { Eligibility } \\
\text { criteria for } \\
\text { ART initiation }\end{array}$ & $\begin{array}{l}\text { Current national } \\
\text { treatment }\end{array}$ & $\begin{array}{l}\text { All HIV-positive individuals who are } \\
\text { aged } 18 \text { years or older, excluding } \\
\text { pregnant or breastfeeding women, } \\
\text { who attend the health facilities will } \\
\text { be offered ART regardless of CD4. }\end{array}$ \\
$\begin{array}{lll}\text { Pre-ART } \\
\begin{array}{l}\text { counseling } \\
\text { sessions }\end{array}\end{array}$ & $\begin{array}{l}\text { 2-3 sessions prior } \\
\text { to ART initiation }\end{array}$ & $\begin{array}{l}\text { Same-day counseling and ART } \\
\text { initiation if patient is ready. Continue } \\
\text { counseling after initiation. }\end{array}$ \\
\hline
\end{tabular}


Laboratory procedures Routine viral load and drug resistance testing are important to understand the implications and impact of the interventions. Patients in both study groups will receive routine viral load and drug resistance testing in addition to the routine standard of care laboratory tests per the recommendation of and as dictated by Swaziland's National Comprehensive HIV Package of Care, including: HIV test; CD4 test, full blood count; AST/ALT; creatinine; and Hepatitis B surface antigen.

Each patient will provide samples every six months for viral load testing. For drug resistance testing, a pretreatment sample will be collected from each patient upon study enrollment and at ART initiation. Upon initial signs of ART failure (two consecutive viral load $>1000$ copies $/ \mathrm{mL}$ ), a post-treatment sample will be collected for drug resistance testing.

Data collection procedures The data for the clinical endpoints will be collected at the facility on an ongoing basis as individuals are enrolled into the study and return for follow-up visits at the facility. Health workers will collect data from individual study participants at each site using the national chronic care file paper-based data collection forms.

Where possible, the study will collect data from existing data sources (i.e. patient's chronic care files, facility registers) to inform the research. Data will also be collected from individual study participants at each visit. For data collected on-site outside of existing Swaziland Ministry of Health procedures, paper-based data collection forms will be used. Two copies of this form are kept: one copy in the patients' clinical file and the second copy as part of the study records.

An electronic database will also be developed to organize all study-related data. Data clerks will be responsible for the daily entry of data from paper forms into the electronic database. All data will be encrypted to ensure patient confidentially before, during, and after the trial. The principal investigator, study statistician, and data manager will have access to the final trial dataset.

Care of patients at the end of the trial Individuals enrolled in this study and initiated on ART will continue to receive care and treatment through Swaziland's public sector health system for the rest of their lives.

\section{Trial outcomes}

A multidisciplinary, mixed-methods approach will be adopted to meet the study aim, including implementation science research, social science research, economic evaluations and cost-effectiveness modeling, and incidence modeling.

\section{Implementation science}

This is one of the first studies in the treatment-asprevention field that has been designed to answer critical implementation questions (i.e. acceptance and retention among patients initiated on ART at higher CD4 thresholds), and determine the "real world" potential of this new prevention intervention [9, 16-19]. In resourcelimited countries, ministries of health need guidance on how to aggressively and effectively adopt the new guidelines and targets, and to understand what the changes will mean for their programs and budgets. As such, this study's primary and secondary outcomes (Table 2) are structured to assess the feasibility, acceptability, clinical outcomes, affordability, and scalability of offering early antiretroviral treatment to all HIV-positive individuals in Swaziland's public sector health system.

Table 2 Primary and secondary outcomes

\begin{tabular}{|c|c|}
\hline Primary outcomes & Definition \\
\hline Retention & $\begin{array}{l}\text { Alive and in care at each 12-month time } \\
\text { point following enrollment }\end{array}$ \\
\hline Viral suppression & $\begin{array}{l}\text { The proportion of individuals alive and in } \\
\text { care whose viral load is below } 1000 \\
\text { copies/mL (virally suppressed) after six } \\
\text { months on treatment }\end{array}$ \\
\hline Secondary outcomes & Definition \\
\hline Retention & $\begin{array}{l}\text { At each six-month time point following } \\
\text { enrollment }\end{array}$ \\
\hline Viral suppression & $\begin{array}{l}\text { At each six-month time point following } \\
\text { enrollment }\end{array}$ \\
\hline Mortality & $\begin{array}{l}\text { At each six-month time point following } \\
\text { enrollment }\end{array}$ \\
\hline $\begin{array}{l}\text { Visit adherence among those } \\
\text { initiated on ART }\end{array}$ & $\begin{array}{l}\text { Proportion of missed visits as a number } \\
\text { of scheduled appointments among } \\
\text { ART-ineligible patients by end } \\
\text { of follow-up }\end{array}$ \\
\hline Drug resistance & $\begin{array}{l}\text { Proportion of drug resistance among } \\
\text { ART-ineligible patients with two } \\
\text { virological failures who have received } \\
\text { genotype resistance testing }\end{array}$ \\
\hline Tuberculosis & $\begin{array}{l}\text { Proportion of ART-ineligible patients } \\
\text { diagnosed with tuberculosis following } \\
\text { enrolment (recurrent and new incident) }\end{array}$ \\
\hline $\begin{array}{l}\text { ART uptake among those } \\
\text { who are eligible }\end{array}$ & $\begin{array}{l}\text { Proportion of HIV-positive individuals } \\
\text { who are eligible for initiation who are } \\
\text { successfully initiated to ART within on } \\
\text { the day of, within two weeks, and one } \\
\text { and three months of becoming eligible }\end{array}$ \\
\hline Cost per patient per year & $\begin{array}{l}\text { Average public and private healthcare } \\
\text { expenditures per patient }\end{array}$ \\
\hline Patient satisfaction & Average patient satisfaction \\
\hline Provider satisfaction & $\begin{array}{l}\text { Average job satisfaction among the } \\
\text { professionals providing HIV treatment }\end{array}$ \\
\hline $\begin{array}{l}\text { Patients' employment, } \\
\text { income, and education }\end{array}$ & $\begin{array}{l}\text { Average patients' employment, income, } \\
\text { and educational attainment }\end{array}$ \\
\hline
\end{tabular}




\section{Social science research}

Social science research methods are used to triangulate the study's findings on ART initiation, adherence, and retention; to help identify effect moderators by analyzing throughout the implementation social and structural factors and differences in health service delivery that may differently affect the uptake of services before and after facilities have transitioned to EAAA; and to contribute to a deeper understanding of reasons for delayed initiation, non-adherence, and non-retention. These objectives will be met by conducting mixed-methods research in a sample of nine study sites including a random quantitative survey, semi-structured interviews with health providers and patients, and participant observation.

The quantitative survey will be conducted with a random sample of patients tested HIV-positive during the control phase and during the intervention phase at six and 12 months (T1, T2, T3, $\mathrm{n}=380$ per survey, 760 in total). The baseline survey will be conducted in 12 months and will include a random sample of HIVpositive patients who have initiated ART less than 12 months prior to the interview. A follow-up survey will be conducted six months after the site's last day of its first transition month with patients who were initiated within six months of the site's transition to the EAAA strategy (intervention stage). The second followup survey at 12 months after the site last day of its first transition months will allow for the comparison of the experiences of those being initiated on ART and adherence before and after the intervention, at six months and at 12 months of being on ART. Changes across time of distal/proximate factors will be studied, including sex, age, family context, socioeconomic status, employment status, health-seeking history, gendered decision-making patterns, and illness experience. Outcomes will include ART initiation, adherence and retention, patients' experience of immediate ART initiation process (i.e. confidentiality, informed consent, enacted stigma, disclosure strategies, and sexual and reproductive health desires).

To assess health providers' experiences and attitudes about EAAA, semi-structured interviews will be conducted with a limited number at two points in time: the second month into the transition and then again four months after the last transition month (information-rich case sampling of those most involved in EAAA). To understand reasons for delayed initiation, non-adherence, and non-retention, qualitative in-depth interviews will be conducted among a diverse sample of $50 \mathrm{HIV}$-positive individuals (ten interviews per category, of which five are men and five are women) who have initiated ART. These interviews will take place at random points of time during the intervention stage at different stages of each individual's cascade of care and treatment.
The categories include EAAA patients who delayed or refused ART initiation and, identified through the EAAA database, patients who are not virally suppressed after six months on treatment, patients who are virally suppressed after six months on treatment, patients who have not been retained in care/on ART at six months, and patients who have been retained in care/on ART at six months.

Finally, at each of the sampled facilities, three weeks of observation will be conducted of pre- and post-test counselling, ART initiation, and adherence counselling: at baseline before the site transitions to the intervention stage, in the second month during transition, and seven months after the first transition month. These observations will take place simultaneously with the baseline patient semi-structured survey interview, health provider, and/or qualitative patient interviews to limit the time spent at each site.

\section{Economic evaluation}

The MaxART study aims to quantify empirically the causal impacts of EAAA on a range of important economic outcomes, including healthcare expenditure, resources use, and patients' welfare. In addition, the cost-effectiveness of the EAAA approach will be estimated through a predictive economic evaluation. To this end, a comprehensive costing study will be conducted, which includes systematic assessments of both the government and private activities for EAAA and the unit costs for these activities. As part of the costing study, the national health sector budgets and expenditure reports will be regularly reviewed and the data relevant to EAAA will be recorded, such as provider salaries and facility, supply chain, drug, and laboratory costs. A timeand-motion study will also be conducted to determine how much time healthcare providers spend on the different HIV treatment-related activities, including consultation time, physical examination, counseling, and interpreting laboratory results. Finally, patient exit interviews will be used to measure private healthcare expenditure and the patient and provider time required under a EAAA protocol.

\section{Incidence modeling}

To inform the national policymaker's interpretation of the stepped-wedge trial, as well as long-term planning of a national EAAA program, a mathematical model has been developed to simulate the heterosexual transmission of HIV and the provision of ART in the catchment area of the study sites and for Swaziland as a whole [20].

The objectives of the HIV incidence modeling component of this study are twofold. First, a narrow, datadriven simulation study will be conducted to estimate the impact of the EAAA stepped-wedge trial on HIV 
incidence during the study period in the catchment area of the study sites. Second, a larger, projection study will be conducted to estimate the impact of a national EAAA program on HIV incidence over three time horizons: (1) five years, (2) ten years, and (3) 15 years (Oct 2016-Sept 2031) for the entire country of Swaziland.

Outcome indicators for both analyses are the incidence rate ratio, number of HIV infections averted, the number of HIV-negative life-years gained, the number of life-years gained, and the number of additional personyears of ART provided (only for the national-level analysis). Outcome indicators for the first analysis will be calculated using model output from both the control and EAAA phases of the clinics, weighted for the population size of the clinic catchment area and the amount of time spent in each phase. Besides population-average outcome indicators, the outcome indicators stratified by age group and gender will also be calculated.

For the estimation of the impact of a national EAAA program on HIV incidence, two scenarios will be contrasted against one another. In the control scenario, the country will maintain its current national treatment guidelines for the entire simulation period. In the EAAA scenario, a shift to universal treatment will be simulated from October 2016 onwards. The rates of HIV diagnosis, ART initiation, viral suppression, and program retention among HIV-positive patients with CD4 cell counts $>500 / \mathrm{mL}$ in the intervention scenario will be equal to those inferred from the narrow, datadriven simulation study.

The epidemiological model will be calibrated to historic data on key demographics (population growth rate, crude birth and death rates, geographical population density), historic HIV prevalence data (stratified by age group and gender), historic ART program size data (number of people on ART, by age group and gender), and recent HIV incidence data, by age group and gender [13]. As parameters for the sexual behavior in the model population are typically difficult to estimate directly from empirical data, due to selection bias and social desirability bias in sexual behavior surveys, these model parameters will be inferred through an iterative Approximate Bayesian Computation algorithm with wide prior distributions [21].

Once the "model world" is deemed sufficiently resembling of the real world, the "model trial" will be calibrated to the real trial by adjusting the hazard functions for initiating ART and dropping out of the ART program in order to match key summary statistics: the size of the ART patient population by clinic, age group, gender, baseline viral load, and baseline CD4 cell count. In addition, the percentage of patients that is virally suppressed six or more months after ART initiation will be matched across all sites, during the period that the sites were offering EAAA, as well as the percentage of patients that are virally suppressed across all sites, during the period that the sites were in the control phase.

\section{Analysis}

The primary analysis will follow the intent to treat approach, with each patient's intervention status assigned as the one in place at their facility at the time of study enrollment. The log rank test, stratified by step time, will be used to assess the statistical significance of any differences observed in one-year retention and six-month suppression rates between the standard of care and early access groups. Kaplan-Meier curves will be produced and used to obtain point and interval estimates of the cumulative incidence of the two primary endpoints at the landmark time points. In the retention analysis, censoring will occur only by administrative end of follow-up or one year from enrollment, whichever happens first. In the viral suppression analysis, only patients initiated to ART who have at least six months of follow-up at the time of administrative end of follow-up and at least one viral load measured after that time will be included. Thus, this endpoint will be interpreted as viral suppression given comprehensive clinical retention.

Censoring for this endpoint occurs at the minimum of the date of the last viral load six months after ART initiation, the administrative end of follow-up, and date of death, whichever happens first. Secondary analysis of the primary endpoints will include estimation of relative risks, their $95 \%$ confidence intervals, and $p$ values for the intervention effects using the Cox proportional hazards model, adjusting for potential measured confounders, including facility, sex, age, BMI, disclosure to partner, occupation, education, baseline CD4, baseline viral load, date of first HIV-positive test, baseline opportunistic infections, baseline WHO stage, and baseline tuberculosis history. Additional analysis will adjust for those variables which are repeatedly updated over time as time-varying covariates, and will, in addition, consider patient's intervention status as time-varying, allowing for the transition of facilities over time, as well as a timevarying eligibility status, allowing for changes in national guidelines and patient preferences.

Marginal structural models will be explored to adjust for possible bias due to dependent censoring [22-24]. Modification of the intervention effects by age, sex, baseline disease stage, facility type, and other hypothesized modifiers will be explored by testing for interaction using the partial likelihood ratio test and providing point and interval estimates of stratum-specific intervention effects. Longitudinal analysis of continuous variables such as BMI, CD4, and viral load in relation to differences in the trajectories of change in these variables over time by intervention group will be conducted under the 
generalized estimating equations framework, following similar modeling strategies as described above [24].

Since one of our primary outcomes is retention, where attrition can be considered the opposite of retention, the intent to treat analysis is ideally situated to assess the impact of the intervention on retention/attrition and cannot be biased by it. However, six-month viral suppression can be biased by differential non-retention. Therefore, in secondary analysis, as recommended by Little et al. [25], we will conduct a sub-study of non-retained clients to better understand the reasons for this and to improve their classification as dead, transferred, or truly lost to follow-up for planned secondary analyses.

\section{Discussion}

The MaxART trial is designed to better understand the "real world" complexities of implementing this treatment for all approach. The quantitative and qualitative social science research produced from this study will be essential to understand the social and behavioral impact of offering ART to HIV-positive individuals soon after diagnosis. Community perception of the intervention and research on how to best engage communities to support this intervention will have an important influence on uptake, linkages to care, retention, and adherence. Further, a comprehensive economic evaluation to assess how expanding access to treatment will improve livelihoods and productivity and, ultimately, decrease the cost to the health system as a whole. Modeling will be used to also understand the cost-effectiveness of the intervention, which is key evidence for the Government of Swaziland's national financial planning. This study has been designed to answer the critical implementation questions posed by the Government of Swaziland as the country works towards rolling out a treatment for all approach across the country to turn the tide on their epidemic.

\section{Trial status}

This trial is underway. Clients will be enrolled into the study through August 2017.

\section{Additional file}

Additional file 1: SPIRIT Checklist MaxART Trial. SPIRIT checklist for the MaxART EAAA Trial, indicating which manuscript page contains each element of the study protocol. (DOCX $53 \mathrm{~kb}$ )

\section{Abbreviations}

3TC: Lamivudine; AIDS: Acquired immunodeficiency syndrome; ART: Antiretroviral treatment; CAB: Community Advisory Board; CD4: Cluster of differentiation 4; DSMB: Data Safety and Monitoring Board; EAAA: Early Access to ART for All; EFV: Efavirenz; FTC: Emtricitabine; HIV: Human immunodeficiency virus; LPV/ r: Lopinavir/ritonavir; MOH: Ministry of Health; SNAP: Swaziland National AIDS Program; TB: Tuberculosis; TDF: Tenofovir; VL: Viral load; WHO: World Health Organization

\section{Acknowledgements}

The MaxART Consortium would like to thank the health workers and community members in Swaziland for their participation in and support of the implementation of the MaxART EAAA trial.

\section{Funding}

We are grateful for the support of the Dutch Postcode Lottery in the Netherlands, the Embassy of the Kingdom of the Netherlands in South Africa/Mozambique, British Columbia Centre of Excellence in Canada, Mylan, and Médecins Sans Frontières. The funders had no role in study design, data collection and analysis, decision to publish, or preparation of the manuscript.

\section{Availability of data and materials}

The trial data will be shared with the Swaziland National Health Research Board, which has a process in place for managing public requests for access to the full protocol, participant-level anonymized dataset, and the statistical code.

\section{Trial oversight}

The Primary Research Team (PRT) comprises the Principal Investigator and Co-Investigators and is responsible for the conduct of the trial and its ethical compliance. The PRT serves as the main decision body for all scientific and administrative issues to ensure the correct implementation of the study protocol.

A Community Advisory Board has been established for communities to protect community interests, including providing substantive input into all aspects of this trial in accordance with community values, culture, and social practices. An independent Data and Safety Monitoring Board (DSMB) has been established to periodically review and evaluate the accumulated study data for participant safety, study conduct and progress, and make recommendations to the MaxART research team on the continuation, modification, or termination of the study. In addition, the trial team will also notify DSMB of all protocol violations and deviations for review.

The objective of this study is to inform the policy decisions of the Government of the Kingdom of Swaziland, and therefore the main priority at the completion of the study will be to prepare the final results for policymakers' review. To support broader dissemination, the findings of this trial will also be shared through presentations and publication in peer-reviewed journals irrespective of the nature of the outcomes of the research. Authorship eligibility guidelines will be followed.

\section{Authors' contributions}

VO is the principal investigator and VO and SM supervised the trial protocol development. FW developed the protocol; DS, TB, and FW contributed to the statistical trial design. All authors participated in the design and coordination of the trial. FW drafted the paper; TB, WD, CL, CM, DS, RR, YF, GK, and MZ contributed to editing the paper. All authors read and approved the final manuscript

\section{Ethics approval and consent to participate}

The trial was approved by the Swaziland National Health Research Review Board in July 2014 (Reference Number: MH/599C/FWA 000 15267). All participants must be able and willing to give verbal consent for trial participation. The final report will follow the CONSORT reporting guidelines for cluster trials [26] and the recommendations for reporting stepped-wedge trials proposed by Hemming et al. [27].

Consent for publication

Not applicable.

\section{Competing interests}

The authors declare that they have no competing interests.

\section{Publisher's Note}

Springer Nature remains neutral with regard to jurisdictional claims in published maps and institutional affiliations.

\section{Author details}

${ }^{1}$ Clinton Health Access Initiative, Boston, MA, USA. ${ }^{2}$ Harvard T.H. Chan School of Public Health, Boston, MA, USA. ${ }^{3}$ Africa Health Research Institute (AHRI), Mtubatuba, South Africa. ${ }^{4}$ Institute of Public Health, University of Heidelberg, Heidelberg, Germany. ${ }^{5}$ The South African Department of Science and 
Technology - National Research Foundation (DST-NRF) Centre of Excellence in Epidemiological Modelling and Analysis (SACEMA), Stellenbosch University, Stellenbosch, South Africa. ${ }^{6}$ Hasselt University, Center for Statistics, Diepenbeek, Belgium. ${ }^{7}$ Ghent University, International Centre for Reproductive Health, Gent, Belgium. ${ }^{8}$ KU Leuven, Rega Institute for Medical Research, Leuven, Belgium. ${ }^{9}$ aidsfonds, Amsterdam, The Netherlands.

${ }^{10}$ Swaziland National Network of People Living with HIV/AIDS (SWANNEPHA), Mbabane, Swaziland. ${ }^{11}$ Clinton Health Access Initiative, Mbabane, Swaziland. ${ }^{12}$ Ministry of Health, Mbabane, Swaziland. ${ }^{13}$ University of Amsterdam, Amsterdam, The Netherlands. ${ }^{14}$ Leiden University Medical Center, Leiden, The Netherlands. ${ }^{15}$ Children's Institute, University of Cape Town, Cape Town, South Africa. ${ }^{16}$ SAfAIDS, Harare, Zimbabwe.

Received: 21 November 2016 Accepted: 27 July 2017

Published online: 18 August 2017

\section{References}

1. UNAIDS. Report on the Global HIV/AIDS Epidemic. Geneva: UNAIDS; 2000. http://data.unaids.org/pub/Report/2000/2000_gr_en.pdf. Accessed 17 July 2016.

2. UNAIDS. Global AIDS Update. Geneva: UNAIDS; 2016. http://www.unaids. org/en/resources/documents/2016/Global-AIDS-update. Accessed 17 July 2016

3. UNAIDS. Fast-track update on investments needed in the AIDS response (2016). Geneva: UNAIDS. http://www.unaids.org/en/resources/documents/ 2016/unaids fast-track update investments needed. Accessed 1 Apr 2016.

4. Government of South Africa. Minister Aaron Motsoaledi: Health Department Budget Vote 2016/2017. 2016. http://www.gov.za/speeches/ debate-health-budget-vote-national-assembly-10-may-2016-dr-aaronmotsoaledi-minister-health. Accessed 17 July 2016.

5. Atun R, Chang AY, Ogbuoji O, Silva S, Resch S, Hontelez J, et al. Longterm financing needs for HIV control in sub-Saharan Africa in 20152050: a modelling study. BMJ Open. 2016;6:e009656. doi:10.1136/ bmjopen-2015-009656.

6. Cohen MS, Chen YQ, McCauley M, Gamble T, Hosseinipour MC, Kumarasamy $\mathrm{N}$, et al. Prevention of HIV-1 infection with early antiretroviral therapy. N Engl J Med. 2011:365:493-505.

7. Lundgren JD, Babiker AG, Gordin F, Emery S, Grund B, Sharma S, et al. Initiation of antiretroviral therapy in early asymptomatic HIV infection. N Engl J Med. 2015;373:795-807. doi:10.1056/NEJMoa1506816.

8. World Health Organization. Consolidated guidelines on the use of antiretroviral therapy for treating and preventing HIV infection: guidelines for a public health approach. Geneva: WHO; 2015.

9. Iwuji CC, Orne-Gliemann J, Tanser F, Boyer S, Lessells RJ, Lert F, et al. Evaluation of the impact of immediate versus WHO recommendationsguided antiretroviral therapy initiation on HIV incidence: the ANRS 12249 TasP (Treatment as Prevention) trial in Hlabisa sub-district, KwaZulu-Natal, South Africa: study protocol for a cluster randomized controlled trial. Trials. 2013;14:1-15. doi:10.1186/1745-6215-14-230.

10. Imuji C, Orne-Gliemann J, Balestre E, Larmarange J, Thiebaut R, Tanser F, et al. Abstract: The impact of universal test and treat on HIV incidence in a rural South African population: ANRS 12249 TasP trial, 2012-2016. In: International AIDS Conference 2016, Durban, South Africa. Durban; 2016 http://programme.aids2016.org/Abstract/Abstract/10537.

11. World Health Organization. Consolidated guidelines on the use of antiretrovira drugs for treating and preventing HIV infection Recommendations for a public health approach. 2nd ed. Geneva: WHO; 2016.

12. Kingdom of Swaziland. Ministry of Health. National comprehensive HIV package of care for adults and adolescents in Swaziland. 2010. http://www. infocenter.nercha.org.sz/sites/default/files/Swaziland\%20Integrated\%20HIV \%20Management\%20Guidelines_0.pdf. Accessed 17 July 2016.

13. Bicego GT, Nkambule R, Peterson I, Reed J, Donnell D, Ginindza H, et al. Recent patterns in population-based HIV prevalence in Swaziland. PLoS One. 2013;8:e77101.

14. Kingdom of Swaziland. Ministry of Health. Annual HIV Programs Report 2015.

15. Hussey MA, Hughes JP. Design and analysis of stepped wedge cluster randomized trials. Contemp Clin Trials. 2007;28:182-91.

16. Hayes R, Ayles H, Beyers N, Sabapathy K, Floyd S, Shanaube K, et al. HPTN 071 (PopART): rationale and design of a cluster-randomised trial of the population impact of an HIV combination prevention intervention including universal testing and treatment - a study protocol for a cluster randomised trial. Trials. 2014:15:57.

17. BCCP: Botswana Combination Prevention Project. clinicaltrials.gov. 2015. https://clinicaltrials.gov/ct2/show/NCT01965470. Accessed 1 Sept 2016.

18. SEARCH: Sustainable East Africa Research in Community Health. clinicaltrials. gov. 2015. https:/clinicaltrials.gov/ct2/show/NCT01864603?term=search +uganda\&rank=1. Accessed 1 Sept 2016.

19. Orne-Gliemann J, Larmarange J, Boyer S, Iwuji C, McGrath N, Bärnighausen $\mathrm{T}$, et al. Addressing social issues in a universal HIV test and treat intervention trial (ANRS 12249 TasP) in South Africa: methods for appraisal. BMC Public Health. 2015;15:209. doi:10.1186/s12889-015-1344-y.

20. Liesenborgs J, Meng F, Hens N DW. Simpact Cyan 0.19. http://www.simpact. org/how-simpact-works/2016. Accessed 5 Aug 2016

21. Delva W, Leventhal GE, Helleringer S. Connecting the dots: network data and models in HIV epidemiology. AIDS. 2016;30:2009-20.

22. Hernan MA, Brumback B, Robins JM. Marginal structural models to estimate the causal effect of zidovudine on the survival of HIV-positive men. Epidemiology. 2000;11:561-70.

23. Rotnitzky A, Robins JM. Semiparametric regression estimation in the presence of dependent censoring. Biometrika. 1995;82:805-20.

24. Fitzmaurice GM, Laird NM, Ware JH. Applied longitudinal analysis. XX: Wiley, New Jersey. 2004.

25. Little RJ, D'Agostino R, Cohen ML, Dickersin K, Emerson SS, Farrar JT, et al. The prevention and treatment of missing data in clinical trials. N Engl J Med. 2012;367:1355-60. doi:10.1056/NEJMsr1203730.

26. Campbell MK, Piaggio G, Elbourne DR, Altman DG. Consort 2010 statement: extension to cluster randomised trials. BMJ. 2012;345(1): e5661. doi:10.1136/bmj.e5661.

27. Hemming K, Haines TP, Chilton PJ, Girling AJ, Lilford RJ. The stepped wedge cluster randomised trial: rationale, design, analysis, and reporting. BMJ. 2015;350:h391.

\section{Submit your next manuscript to BioMed Central and we will help you at every step:}

- We accept pre-submission inquiries

- Our selector tool helps you to find the most relevant journal

- We provide round the clock customer support

- Convenient online submission

- Thorough peer review

- Inclusion in PubMed and all major indexing services

- Maximum visibility for your research

Submit your manuscript at www.biomedcentral.com/submit
) Biomed Central 